\title{
Leakage and lysis of lipid membranes induced by the lipopeptide surfactin
}

\author{
Heiko Heerklotz $\cdot$ Joachim Seelig
}

Received: 15 May 2006/Revised: 11 August 2006/Accepted: 25 August 2006/ Published online: 19 October 2006

(C) EBSA 2006

\begin{abstract}
Surfactin is a lipopeptide produced by Bacillus subtilis which possesses antimicrobial activity. We have studied the leakage and lysis of POPC vesicles induced by surfactin using calcein fluorescence de-quenching, isothermal titration calorimetry and ${ }^{31} \mathrm{P}$ solid state NMR. Membrane leakage starts at a surfactin-to-lipid ratio in the membrane, $R_{\mathrm{b}} \approx 0.05$, and an aqueous surfactin concentration of $C_{\mathrm{S}}^{\mathrm{w}} \approx 2 \mu \mathrm{M}$. The transient, graded nature of leakage and the apparent coupling with surfactin translocation to the inner leaflet of the vesicles, suggests that this low-concentration effect is due to a bilayer-couple mechanism. Different permeabilization behaviour is found at $R_{\mathrm{b}} \approx 0.15$ and attributed to surfactin-rich clusters, which can induce leaks and stabilize them by covering their hydrophobic edges. Membrane lysis or solubilization to micellar structures starts at $R_{\mathrm{b}}^{\mathrm{sat}}=0.22$ and $C_{\mathrm{S}}^{\mathrm{w}}=9 \mu \mathrm{M}$ and is completed at $R_{\mathrm{m}}^{\mathrm{sol}}=0.43$ and $C_{\mathrm{S}}^{\mathrm{w}}=11 \mu \mathrm{M}$. The membrane-water partition coefficient of surfactin is obtained as $K=2 \times 10^{4} \mathrm{M}^{-1}$. These data resolve inconsistencies in the literature and shed light on the variety of effects often referred to as detergent-like effects of antibiotic peptides on membranes. The results are compared with published parameters characterizing the hemolytic and antibacterial activity.
\end{abstract}

Dedicated to Prof. K. Arnold on the occasion of his 65th birthday.

H. Heerklotz $(\bowtie) \cdot J$. Seelig

Biozentrum, University of Basel, Klingelbergstr. 70,

4056 Basel, Switzerland

e-mail: heerklotz@gmx.net
Keywords Antibiotic peptide - Membrane lysis . Membrane permeabilization - Asymmetric partitioning $\cdot$ Calcein leakage

\section{Introduction}

Surfactin is a mixture of closely related lipopeptides produced by Bacillus subtilis. It consists of a cyclic heptapeptide moiety with the typical sequence GluLeu-D-Leu-Val-Asp-D-Leu-Leu which is closed to a lactone ring by a $\mathrm{C}_{14-15} \beta$-hydroxy fatty acid. Surfactin is highly surface active, which may play a key role for the motility of the bacillus (Kinsinger et al. 2003) as well as for technical applications (Cameotra and Makkar 2004). Given the great interest in the development of new peptide antibiotics, it is of particular relevance that surfactin possesses also hemolytic (Kracht et al. 1999), anti-viral (Kracht et al. 1999; Vollenbroich et al. 1997a), anti-bacterial (Beven and Wroblewski 1997; Vollenbroich et al. 1997b), and anti-tumor (Kameda et al. 1974) activity. It is generally believed that these properties result from its capability of permeabilizing cellular membranes and viral envelopes.

A variety of models and potential mechanisms have been proposed for peptide-induced membrane permeabilization, one of which has been referred to as "detergent-like". This term is used for an unspecific effect that does not involve specific oligomers forming a pore. Such a behavior can be recognized by the lack of a distinct size threshold for permeating solutes (Ladokhin and White 2001). Detergent-like leaks were also identified by allowing for a fast lipid flip-flop of the lipids across the bilayer, in contrast to pore-forming oligomers, (Lohner and Epand 1997). For surfactin, a 
strong detergent-like perturbation of the membrane has been found comparing the energies of self-association and membrane partitioning (Heerklotz and Seelig 2001). Deuterium NMR has revealed that the hydrophobic residues of the peptide moiety penetrate rather deeply into the membrane interface, where they may induce a stronger destabilization than typical detergents (Heerklotz et al. 2004). Dufour et al. (2005) have shown by comparing cyclic with linear surfactin analogs that the bulky nature of the cyclic peptide group is a prerequisite for membrane permeabilization but not for surface activity, as expected for detergentlike behaviour. The proposal that membranes are permeabilized by dimers of surfactin (Carrillo et al. 2003) argues, however, in favor of a more specific mechanism.

Detergents can permeabilize membranes by different mechanisms: (1) the bilayer-couple model (Sheetz and Singer 1974) describes the effect of detergents that cannot flip quickly from the outer to the inner monolayer. Their insertion into the outer monolayer leads to an asymmetric increase in lateral pressure that tends to bend the bilayer and may lead to a transient disruption of the membrane at a certain threshold (Heerklotz 2001). (2) At higher detergent concentration, detergent-detergent contacts or, more generally, detergentrich clusters in the membrane, become abundant, which are highly destabilizing of the lamellar structure and which can stabilize leaks by covering their edges by a detergent-rich rim. (3) At a characteristic detergentto-lipid ratio in the membrane, $R_{\mathrm{b}}^{\text {sat }}$, the lipid membrane starts to become solubilized to mixed micelles.

For surfactin, the mole ratio at the onset of solubilization was published to be 0.92 (Carrillo et al. 2003). This is at variance with preliminary results and theoretical predictions from partitioning experiments $\left(R_{\mathrm{b}}^{\text {sat }} \approx 0.2\right)$ (Heerklotz and Seelig 2001), and would argue strongly against a detergent-like mode of action of surfactin, since values of $R_{\mathrm{b}}^{\text {sat }} \approx 1$ are typical for rather weak membrane perturbants (see for a compilation Heerklotz and Seelig 2000b, 2001). Weak detergents are active only in very high concentrations, which are unrealistic for a biological system. Potent synthetic detergents such as Triton X-100 (Heerklotz 2002; Paternostre et al. 1988) or $\mathrm{C}_{12} \mathrm{EO}_{8}$ (Edwards and Almgren 1991; Heerklotz et al. 1996) solubilize membranes at about $R_{\mathrm{b}}^{\text {sat }} \approx 0.6$ or even less, depending on lipid species and temperature.

Here, we measure $R_{\mathrm{b}}^{\text {sat }}$ using isothermal titration calorimetry (for a review, cf. Heerklotz and Seelig 2000b). The result is further checked by ${ }^{31} \mathrm{P}$ NMR. The available membrane leakage experiments with surfactin specify apparent leakage rates at very high surfactin concentrations (Carrillo et al. 2003). To shed light on the physiologically more interesting non-lytic membrane permeabilization, we have performed leakage experiments at much lower concentrations. Our results suggest a sequence of detergent-like permeabilization effects with increasing concentration. These findings are of general interest for understanding the actions of antimicrobial peptides on membranes.

\section{Materials and methods}

\section{Materials}

Surfactin was purchased from Sigma, St. Louis, USA and the lipid 1-palmitoyl-2-oleoyl-sn-glycero-3-phosphocholine (POPC) was from Avanti Polar Lipids, Alabaster AL, USA. Calcein, Tris buffer, EDTA and $\mathrm{NaCl}$ were from Fluka, Buchs, Switzerland.

\section{Isothermal titration calorimetry}

Isothermal titration calorimetry was performed on a VP ITC system from MicroCal (Northampton, USA) (Chellani 1999). The ITC solubilization assay is described elsewhere (Heerklotz et al. 1995; Heerklotz and Seelig 2000b; Wenk and Seelig 1997). Briefly, the $300 \mu \mathrm{L}$ injection syringe is filled with a micellar solution of surfactin. About $1.5 \mathrm{~mL}$ of a lipid vesicle suspension is loaded into the calorimeter cell and a series of injections is performed. The heat peaks recorded after each injection are evaluated using the instrument software.

For the preparation of unilamellar vesicles with an approximate diameter of $100 \mathrm{~nm}$, the buffer $(10 \mathrm{mM}$ Tris, $100 \mathrm{mM} \mathrm{NaCl}, 1 \mathrm{mM}$ EDTA, $\mathrm{pH} 8.5$ ) was added to the dry lipid, the suspension was vortexed and subjected to five freeze-thaw cycles. Then, the material was extruded by 11 passages through 2 stacked $\mathrm{Nu}$ clepore $^{\mathrm{TM}}$ polycarbonate membranes of $100 \mathrm{~nm}$ pore size in a homebuilt miniextruder (MacDonald et al. 1991).

\section{Leakage assay}

Vesicle leakage was assessed from de-quenching of the water-soluble, membrane-impermeable dye calcein (Allen and Cleland 1980; Wieprecht et al. 1997).

Vesicles were prepared by extrusion as explained above in a buffer containing $70 \mathrm{mM}$ calcein, $10 \mathrm{mM}$ Tris, $1 \mathrm{mM}$ EDTA at $\mathrm{pH}$ 8.5. This vesicle suspension was subject to gel filtration using a Sephadex G75 column in order to replace the calcein buffer outside 
the vesicles with $100 \mathrm{mM} \mathrm{NaCl}, 10 \mathrm{mM}$ Tris, $1 \mathrm{mM}$ EDTA, $\mathrm{pH}$ 8.5. The lipid concentration was determined by a phosphorus assay and the various preparations yielded lipid concentrations slightly above $6 \mathrm{mM}$. The vesicle suspension was diluted with calceinfree buffer to $6 \mathrm{mM}$.

For determining the concentration-dependent leakage after $1 \mathrm{~h}$, series with $50 \mu \mathrm{L}$ (A), $190 \mu \mathrm{L}$ (B), $590 \mu \mathrm{L}(\mathrm{C})$, and $1,990 \mu \mathrm{L}$ (D) of surfactin solutions in calcein-free buffer were prepared. Each series typically included ten samples with varying surfactin concentrations ranging from 0 to $210 \mu \mathrm{M}$ (A), 0 to $70 \mu \mathrm{M}$ (B), 0 to $30 \mu \mathrm{M}(\mathrm{C})$, and 0 to $15 \mu \mathrm{M}(\mathrm{D})$. The process was started by adding $10 \mu \mathrm{L}$ of $6 \mathrm{mM}$ lipid vesicle solution (with entrapped calcein buffer) to each sample, so that the lipid concentrations in the samples were $1 \mathrm{mM}(\mathrm{A})$, $300 \mu \mathrm{M}$ (B), $100 \mu \mathrm{M}$ (C) and $30 \mu \mathrm{M}$ (D). The samples were incubated for $1 \mathrm{~h}$ at $25^{\circ} \mathrm{C}$ in the dark under gentle shaking. After the incubation period, all samples were filled up to a total volume of $2 \mathrm{~mL}$ and a common lipid concentration of $30 \mu \mathrm{M}$. Then, the fluorescence intensity, $F$, was measured in a JASCO FP 777 spectrofluorometer. Finally, $200 \mu \mathrm{L}$ of $100 \mathrm{mM}$ Triton X-100 were added to each sample in order to completely solubilize the vesicles and the intensity after releasing all calcein, $F_{\max }$, was measured.

The quenching factor, $Q$, was calculated on the basis of the fluorescence intensity of surfactin-free samples prior to $\left(F_{0}\right)$ and after lysis $\left(=0.9 \cdot F_{\max }\right.$, correcting for the dilution of the dye by the Triton solution):

$Q=\frac{F_{0}}{F_{\max }}$,

amounting to $Q=7-10$ for different vesicle preparations, depending on the efficacy of the removal of free calcein upon gel filtration. The degree of dequenching, $F_{\text {rel }}$, is defined as:

$F_{\text {rel }}=\frac{F-F_{0}}{F_{\max }-F_{0}}$

and ranges from 0 to $1(100 \%)$. Each series was repeated 2-4 times, partially with separately prepared vesicles.

This protocol avoids a number of pitfalls. The buffers inside and outside the vesicles are isosmotical. The sample is kept free from organic solvents. Small volumes of the lipid suspension are injected into large volumes of surfactin solutions so that the local surfactin concentrations during mixing are only slightly above the average value. A highly concentrated stock solution of surfactin might permeabilize lipid vesicles during the mixing process, although the average surfactin concentration is not sufficient for inducing leakage. In the spectrometer, all samples contain the same amount of lipid vesicles at very low concentration so that turbidity effects are small and equal. All samples showing the same degree of de-quenching share the same free and entrapped calcein concentrations, so that also inner filter effects are the same. During incubation, different lipid concentrations are used in order to be able to correct for surfactin that does not bind to the membrane (cf. below) and as a test of consistency.

\section{P NMR}

Solid-state ${ }^{31} \mathrm{P}$ NMR was measured on a Bruker DRX $400(9.4 \mathrm{~T})$ at $161.98 \mathrm{MHz}$. A pulse-and-acquire program with proton decoupling was used with a $90^{\circ}$ pulse of $5 \mu \mathrm{s}$ and a repetition time of $4 \mathrm{~s}$ to avoid saturation, 300-400 FID's were accumulated and a line broadening of $150 \mathrm{~Hz}$ was applied.

The measurements were carried out with POPC partially deuterated at the $\mathrm{C}_{9}$ and $\mathrm{C}_{10}$ position of the oleic acid chain to allow for simultaneous deuterium NMR experiments as shown elsewhere (Heerklotz et al. 2004). For each sample, $\sim 10 \mathrm{mg}$ of lipid and appropriate amounts of surfactin were mixed in dichloromethane, dried by a gentle stream of nitrogen followed by vacuum over night, and suspended in $100 \mu \mathrm{L}$ of buffer (110 mM Tris, 1 mM EDTA, pH 8.5) to form multilamellar vesicles.

\section{Theory}

The local surfactin-to-lipid mole ratio within the membrane, $R_{\mathrm{b}}$, determines the properties of the membrane and is given as:

$R_{\mathrm{b}}=\frac{C_{\mathrm{S}}^{\mathrm{b}}}{C_{\mathrm{L}}}$

where $C_{\mathrm{S}}^{\mathrm{b}}$ denotes the concentration of membranebound detergent and $C_{\mathrm{L}}$ the lipid concentration.

The mole ratio partition coefficient, $K$, is defined as

$K \equiv \frac{R_{\mathrm{b}}}{C_{\mathrm{S}}^{\mathrm{w}}}=\frac{C_{\mathrm{S}}^{\mathrm{b}}}{C_{\mathrm{L}} C_{\mathrm{S}}^{\mathrm{w}}}$,

where $C_{\mathrm{S}}^{\mathrm{w}}$ stands for the concentration of aqueous detergent monomers. An advantage of this definition is that $K$ has been found to be virtually constant for many surfactants, independent of their membrane content $R_{\mathrm{b}}$ 
(Heerklotz and Seelig 2000b). A partition coefficient between the aqueous solution and mixed micelles can be defined analogously.

Using the conservation of mass, $C_{\mathrm{S}}=C_{\mathrm{S}}^{\mathrm{b}}+C_{\mathrm{S}}^{\mathrm{w}}$ (in the absence of micelles) and Eqs. 3 and 4 we obtain:

$C_{\mathrm{S}}=R_{\mathrm{b}} \cdot C_{\mathrm{L}}+C_{\mathrm{S}}^{\mathrm{w}}=R_{\mathrm{b}}\left(C_{\mathrm{L}}+\frac{1}{K}\right)$.

Equation 5 has been widely used to determine the characteristic membrane composition, $R_{\mathrm{b}}$, that leads to a certain membrane property, such as the onset of solubilization $\left(R_{\mathrm{b}}^{\text {sat }}\right)$ (Heerklotz and Seelig 2000b; Lichtenberg et al. 1983), a certain leakage (i.e., $R_{\mathrm{b}}$ (50\% de-quenching), e.g., De la Maza and Parra 1994; Encinas and Lissi 1982), a characteristic generalized fluorescence polarization (Heerklotz et al. 1994), etc. Total surfactant concentrations, $C_{\mathrm{S}}$, corresponding to the same membrane property (i.e., sharing the same $R_{\mathrm{b}}$ ) are determined at different lipid concentrations, $C_{\mathrm{L}}$. A plot of such $C_{\mathrm{S}}\left(C_{\mathrm{L}}\right)$ allows determining $R_{\mathrm{b}}, C_{\mathrm{S}}^{\mathrm{w}}$, and $K$ by a linear regression according to Eq. 5. It should be noted that the analogous approach holds for the completion of solubilization (superscript sol). A fit of $C_{\mathrm{S}}^{\mathrm{sol}}\left(C_{\mathrm{L}}\right)$ yields $R_{\mathrm{m}}^{\mathrm{sol}}$, the minimum local surfactin-tolipid mole ratio within mixed micelles, the corresponding aqueous concentration $C_{\mathrm{S}}^{\mathrm{w}, \text { sol }}$, and $K$ for mixed micelles.

\section{Results}

ITC

Figure 1a shows the raw data of an ITC solubilization experiment (Heerklotz et al. 1996; Heerklotz and Seelig 2000b) titrating a $1 \mathrm{mM}$ POPC vesicle suspension with a $1 \mathrm{mM}$ micellar solution of surfactin in $6 \mu \mathrm{L}$ injections. Initially, the surfactin concentration in the cell is small; the injected surfactin micelles dissolve and most of the surfactin is inserted into the lipid vesicles. The accompanying heat peaks are positive, indicating that this micelle-to-bilayer transfer is an endothermic process. The course of the integrated heats of injection is shown as a bold solid line in Fig. 1b after normalization with respect to the injected mole number of surfactin. The abscissa of Fig. 1b is the average of the surfactin-to-lipid molar ratios in the cell before and after a given injection.

At about one surfactin per 20 lipids (mole ratio $R=0.05$ ), the heats of injection reach a local minimum and the response of the system to the injection of surfactin becomes slower. This local minimum is typi-
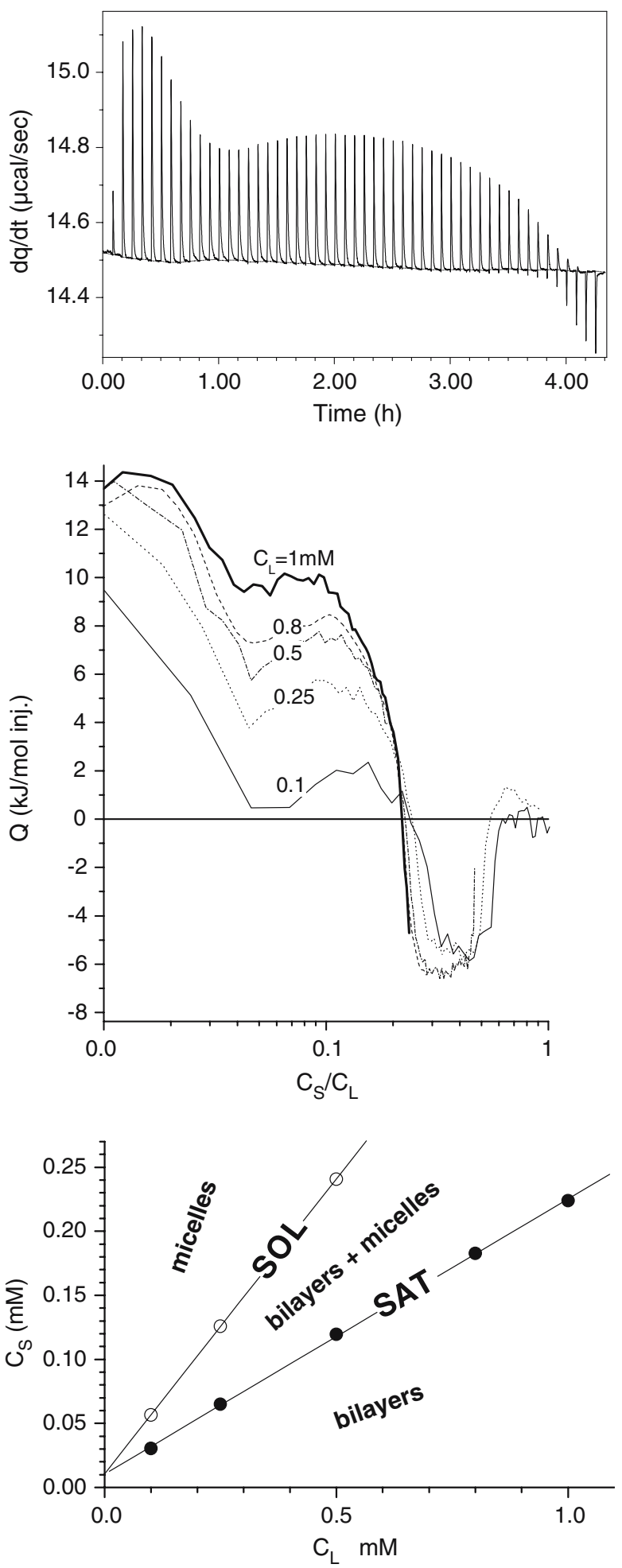

Fig. 1 Results of ITC solubilization experiments titrating POPC vesicles $\left(C_{\mathrm{L}}\right.$ given in the plot) with a $1 \mathrm{mM}$ micellar dispersion of surfactin at $25^{\circ} \mathrm{C}$. a Raw data for $C_{\mathrm{L}}=1 \mathrm{mM}$. b Normalized heats of injection as a function of the total surfactin-to-lipid molar ratio, $C_{\mathrm{S}} / C_{\mathrm{L}}$. c Phase diagram showing $C_{\mathrm{S}}$ at the break points of the titration curve as a function of $C_{\mathrm{L}}$. The parameters of the linear fits according to Eq. 5 are given in Table 1 
Table 1 Characteristic surfactin-to-lipid molar ratios in the membrane, $R_{\mathrm{b}}$, and in micelles, $R_{\mathrm{m}}$, and corresponding aqueous surfactin concentrations, $C_{\mathrm{S}}^{\mathrm{w}}$, giving rise to certain degrees of leakage and the onset (SAT) and completion (SOL) of solubilization

\begin{tabular}{llll}
\hline & $R_{\mathrm{b}}$ & $C_{\mathrm{S}}^{\mathrm{w}}(\mu \mathrm{M})$ & $K\left(\mathrm{M}^{-1}\right)$ \\
\hline Membranes & & & \\
25\% de-quenching & 0.06 & 2.0 & $2 \times 10^{4}$ \\
50\% de-quenching & 0.08 & 2.6 & $3 \times 10^{4}$ \\
75\% de-quenching & 0.11 & 3.0 & $4 \times 10^{4}$ \\
SAT (ITC) & 0.22 & 9 & $2.4 \times 10^{4}$ \\
\hline & $R_{\mathrm{m}}$ & $C_{\mathrm{S}}^{\mathrm{w}}(\mu \mathrm{M})$ & $K\left(\mathrm{M}^{-1}\right)$ \\
\hline Micelles & & & \\
SOL (ITC) & 0.46 & 11 & $4.3 \times 10^{4}$ \\
\hline
\end{tabular}

The parameters fit the data in Figs. $1 \mathrm{c}$ and $4 \mathrm{c}$ according to Eq. 5

cal for molecules that do not flip spontaneously across the membrane within a few minutes and has been attributed to a membrane permeability threshold caused by the bilayer curvature strain in an asymmetrically expanded membrane (Binder and Lindblom 2003; Heerklotz 2001; Hildebrand et al. 2002).

At $C_{\mathrm{S}} / C_{\mathrm{L}}=0.22$, the heat peaks change their sign from endothermic to exothermic, indicating the onset of solubilization by the appearance of stable mixed micelles (Heerklotz et al. 1995; Heerklotz and Seelig 2000b).

Figure $1 \mathrm{~b}$ includes also data from titration experiments with smaller lipid concentrations in the cell. In this case, larger mole ratios are reached in the end of the titration and another breakpoint is found at $C_{\mathrm{S}}^{\text {sol }}$. The heat of titration then returns to values close to zero, indicating the completion of the solubilization process.

With decreasing lipid concentration, the (inactive) aqueous fraction of the peptide gets larger and higher total ratios $C_{\mathrm{S}} / C_{\mathrm{L}}$ are necessary to reach the characteristic values of $R_{\mathrm{b}}^{\text {sat }}$ and $R_{\mathrm{m}}^{\text {sol }}$ within the membranes or micelles, respectively.

Figure 1c shows the surfactin concentrations at which the breakpoints in the titration curves occur as a function of the lipid concentrations. Linear regressions according to Eq. 5 yield parameters collected in Table 1.

\section{${ }^{31} \mathrm{P}$ NMR}

Phosphorus NMR is used as a tool to distinguish between lamellar and micellar phases. In multilamellar vesicles, where the orientations of a molecule with respect to the external field is not averaged within the time scale of the experiment, one obtains a broadened

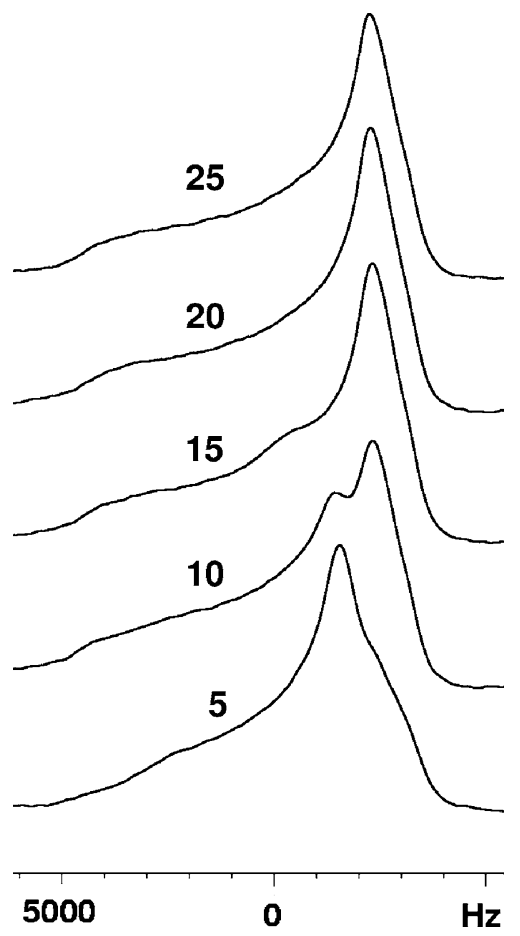

Fig. $2{ }^{31} \mathrm{P}$ NMR spectra of POPC vesicles containing $R_{\mathrm{b}} \approx C_{\mathrm{S}} /$ $C_{\mathrm{L}}=0.2$ moles of surfactin per lipid at various temperatures as specified in ${ }^{\circ} \mathrm{C}$ in the plot. The spectrum at $25^{\circ} \mathrm{C}$ shows the typical powder pattern for the lamellar phase, a narrow signal suggesting the coexistence of micelles appears at $15^{\circ} \mathrm{C}$ and grows upon cooling

spectrum with a powder pattern as shown in Fig. 2 (top spectrum measured at $25^{\circ} \mathrm{C}$ ).

Phospholipids solubilized in micelles show a faster angular re-orientation and yield a spectrum with a substantially smaller chemical shift anisotropy. Figure 2 shows this micellar signal on top of the lamellar pattern in a sample containing surfactin $\left(R_{\mathrm{b}}=0.2\right)$ to emerge at $15^{\circ} \mathrm{C}$ and grow upon further cooling. At $25^{\circ} \mathrm{C}$, no micelles are present at $R_{\mathrm{b}}=0.2$ but a distinct micellar signal is visible at $R_{\mathrm{b}}=0.3$ (cf. spectra included in Fig. 5).

\section{Kinetics of leakage}

Calcein leakage studies monitor an increase in fluorescence intensity that accompanies the release of dye from the vesicle interior (high concentration, strong self-quenching) to the free solution (low concentration, little self-quenching). This 'de-quenching' is non-linearly related to the amount of dye released and to the extent of leakage.

Figure $3 \mathrm{a}$ shows the increase in the fluorescence of previously vesicle-entrapped calcein after treatment with different concentrations of surfactin. The respec- 

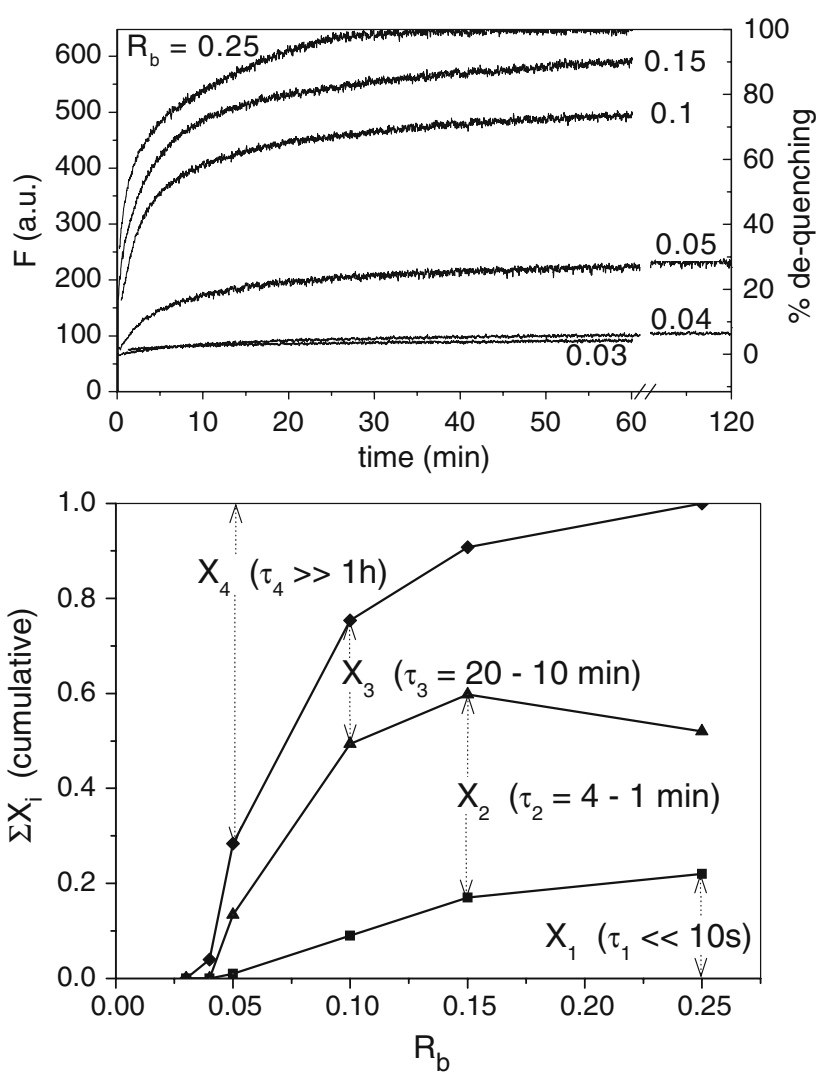

Fig. 3 Kinetics of calcein fluorescence de-quenching induced by different surfactin contents, $R_{\mathrm{b}}$. Top Time-dependent fluorescence intensities, which were modelled by Eq. 6. Bottom Fit results for $X_{1}$ (filled square), $X_{1}+X_{2}$ (filled triangle), $X_{1}+X_{2}+X_{3}$ (filled diamond) (so that the distances between two lines or between lines and frame correspond to $X_{i}$ as indicated by the dotted arrows in the plot) and the corresponding lifetimes $\tau$ (see plot)

tive surfactin-to-lipid molar ratio in the membrane, $R_{\mathrm{b}}$, was calculated on the basis of $K=22 \mathrm{mM}^{-1}$ (Heerklotz and Seelig 2001). Up to $R_{\mathrm{b}}=0.04$ there is hardly any leakage detected. At higher surfactin concentration, complex kinetics of fluorescence de-quenching were observed which could be fitted with a biexponential model:

$$
\begin{aligned}
& F(t)= \\
& \quad F_{0}+\left(F_{\max }-F_{0}\right) \cdot\left[1-X_{4}-X_{3} \exp ^{-t / \tau_{3}}-X_{2} \exp ^{-t / \tau_{2}}\right]
\end{aligned}
$$

For $t \rightarrow 0$, this equation simplifies to:

$F(0)=F_{0}+\left(F_{\max }-F_{0}\right) \cdot X_{1}$

considering an intensity fraction $X_{1}=1-X_{2}-X_{3}-X_{4}$ that is appearing within the dead time of the measurement $(\tau<<10 \mathrm{~s})$. For long times, Eq. 6 becomes:
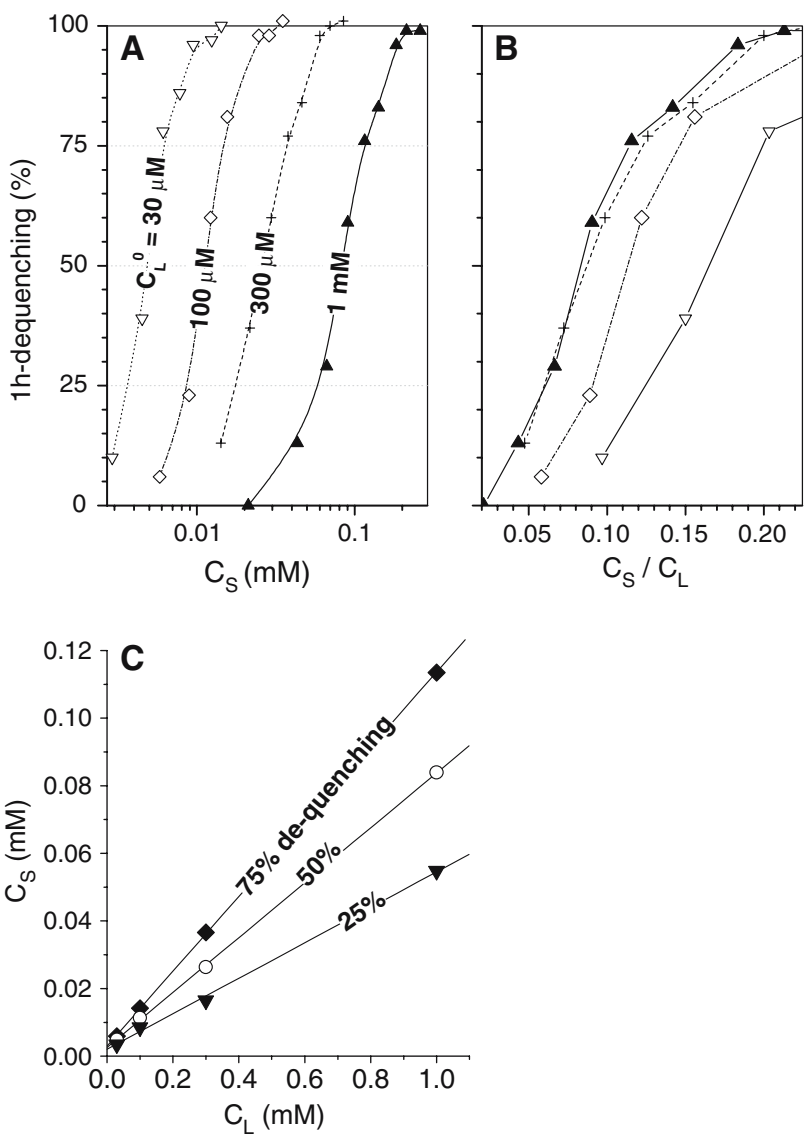

Fig. 4 Surfactin-induced de-quenching of calcein. The relative fluorescence intensity $F_{\text {rel }}$ after $1 \mathrm{~h}$ is shown as a function of the surfactin concentration, $\mathrm{C}_{\mathrm{S}}(\mathbf{a})$ and the mole ratio $C_{\mathrm{S}} / C_{\mathrm{L}}(\mathbf{b})$ for series with lipid concentrations $C_{\mathrm{L}}$ specified in the plot. c $C_{\mathrm{S}}\left(C_{\mathrm{L}}\right)$ giving rise to characteristic values of de-quenching as indicated, linear regression according to Eq. 6 yielded the parameters given in Table 1

$F(\infty)=F_{\max }-\left(F_{\max }-F_{0}\right) \cdot X_{4}$,

where a finite value of $X_{4}$ implies that a fraction of the dye is not released even after several hours.

The fractions $X_{1}-\mathrm{X}_{4}$ are illustrated in Fig. 3 (bottom) in a cumulative plot where, for example, the fraction $X_{2}$ corresponds to the range between the squares and the triangles. Leakage starts at $R_{\mathrm{b}} \sim 0.05$, where $X_{2} \sim 15 \%$ of the maximum de-quenching, $F_{\max }-$ $F_{0}$, appears with $\tau \sim 4 \mathrm{~min}$ and another $15 \%\left(X_{3}\right)$ with $\tau_{3} \sim 20 \mathrm{~min}$. The remaining calcein does not leak out, it corresponds to $X_{4} \sim 70 \%$ of the maximum signal determined by Triton-induced lysis. Generally, leakage is accelerated and its extent is increased with increasing surfactin content, $R_{\mathrm{b}}$. It is important to note that the fractions $X_{i}$ do not represent different leakage mechanisms proceeding in parallel but characteristic fractions of the dye showing different leakage behaviour. 
Membrane annealing after graded leakage

There are two general possibilities to explain these different fractions of the intensity appearing with different rates, an (1) all-or-none mechanism and (2) a graded mechanism (Ladokhin et al. 1995).

1. In the all-or-none case, the different kinetics represent different fractions of vesicles. Some vesicles are leaking with a fast rate and others do not. This may be a consequence of different initial sizes of the vesicles, a heterogeneous distribution of surfactin, or other reasons. It would mean that after, e.g., $45 \mathrm{~min}$, virtually all dye would be released from the leaking vesicle fraction $\left(X_{1}+X_{2}+X_{3}\right)$, whereas a fraction $X_{4}$ of the vesicles would still contain all previously entrapped dye.

2. In the case of graded release, all vesicles release part of the entrapped dye followed by an annealing of the membrane, so that the remaining dye is retained in the interior of the vesicles.

An assay to distinguish between both cases was performed as follows. After separating calcein-loaded vesicles from free calcein by gel filtration, we measured the fluorescence intensity prior to $\left(F_{0}\right)$ and after lysis by Triton (yielding $F_{\max }$ ). The quenching factor, which depends on the concentration of calcein in the interior of the vesicles, amounted to $F_{\max } / F_{0} \approx 9$. Calcein-loaded vesicles were incubated with and without surfactin for $60 \mathrm{~min}$. Then, free calcein was again removed by a second gel filtration. In turn, the quenching factor was measured by comparing the intensities before and after lysis. Whereas the vesicles that were not exposed to surfactin showed a quenching factor of 9 , those that had released some dye (20 and $40 \%$ de-quenching) exhibited a significantly reduced quenching factor of $\approx 5$. This provides strong evidence that the partial dequenching is due to a gradual release of dye from virtually all vesicles followed by an annealing process that prevents further leakage. Thus, the concentration of entrapped dye is reduced and so is the extent of selfquenching. If partial leakage was an all-or-none process, one would expect the quenching factor to be unchanged by surfactin because "empty" vesicles have no effect on the quenching factor.

\section{Concentration dependence of leakage}

After an exposure of the vesicles to surfactin for $45 \mathrm{~min}$, the fast process of dye release is essentially finished and further de-quenching has come to a halt or become a slow process. Therefore, we have chosen an exposure time $1 \mathrm{~h}$ to compare the effects of different surfactin and lipid concentrations on vesicle leakage.

Figure $3 \mathrm{~b}$ shows the $1 \mathrm{~h}$ de-quenching curves obtained for different lipid concentrations as a function of the surfactin concentration. The shift illustrates the simple fact that larger lipid concentrations require also more surfactin to be permeabilized to a given extent. This effect is compensated if the curves are re-plotted as a function of the surfactin-to-lipid molar ratio $C_{\mathrm{S}} / C_{\mathrm{L}}$ (Fig. 4b). The fact that the curves measured at $300 \mu \mathrm{M}$ and $1 \mathrm{mM}$ POPC agree with each other implies that virtually all surfactin is membrane-bound and thus active as a permeabilizer, i.e., $C_{\mathrm{S}} / C_{\mathrm{L}} \sim R_{\mathrm{b}}$ at $C_{\mathrm{L}} \geq 0.3 \mathrm{mM}$. At lower $C_{\mathrm{L}}$, there is a significant amount of surfactin in aqueous solution so that higher $C_{\mathrm{S}}$ are required to reach the $R_{\mathrm{b}}$ corresponding to a certain leakage. Horizontal cuts through Fig. 4a at selected levels of de-quenching (see grid lines in Fig. 4a) yield interpolated values for the total concentrations, $C_{\mathrm{S}}$, leading to a characteristic leakage at a given $C_{\mathrm{L}}$. These values are plotted in Fig. $4 \mathrm{c}$ and linear regressions according to Eq. 5 yield the corresponding values of $R_{\mathrm{b}}, C_{\mathrm{S}}^{\mathrm{w}}$, and estimates for $K$ as listed in Table 1 .

\section{Discussion}

\section{Leakage}

The data suggest characteristic concentration ranges with different membrane permeabilization behaviour. Below a surfactin-to-lipid mole ratio $R_{\mathrm{b}}=0.04$ in the membrane, no leakage was detected and ITC suggests that added surfactin inserts exclusively into the outer leaflet of the membrane.

At concentrations of $R_{\mathrm{b}} \sim 0.05$ to 0.1 , both leakage and surfactin translocation (ITC curve) start simultaneously but leakage is graded and the membrane anneals after a partial dye release. This behaviour is in accord with a bilayer-couple mechanism where strain inducing leakage arises from a selective increase in the area requirement of the outer (but not the inner) leaflet by surfactin. Transient membrane failure allows some surfactin and lipid to flip to the inner leaflet and some calcein to be released from the vesicle interior. Then, the driving force for leakage is eliminated and the membrane anneals. This mechanism also accounts for the observation that membrane permeabilization by surfactin (Carrillo et al. 2003) and other antibiotic peptides (Prenner et al. 2001) is attenuated by cholesterol. First, cholesterol makes membranes mechanically more stable, so that more energy may be required for breaking it. Second, cholesterol can bal- 
ance the asymmetric expansion of the outer leaflet by its ability to undergo a fast flip to the inner leaflet (Lange et al. 1981).

At a higher surfactin content of $R_{\mathrm{b}} \sim 0.1$ to 0.2 , all dye can leak out from the vesicles after sufficient time and the heat of insertion of surfactin into membranes decreases. Phosphorus NMR does, however, show that no micelles are formed at $R_{\mathrm{b}} \leq 0.2$. This behaviour is consistent with another mechanism of membrane permeabilization that is based on strongly destabilizing surfactin-surfactin contacts or surfactin-rich clusters in the membrane. Such clusters of molecules preferring a strongly curved aggregate surface may lead to a leak that is stabilized by a surfactin-rich rim covering its edge. Such leaks may also be transient but they emerge from an equilibrium state and will re-appear with the same probability. Hence, the membrane cannot anneal and all entrapped dye leaks out after some time. The permeabilizing activity of surfactin $(50 \%$ de-quenching of calcein at $\left.R_{\mathrm{b}}=0.08\right)$ is stronger than that of strong detergents such as Triton (50\% de-quenching of carboxyfluorescein at $R_{\mathrm{b}} \sim 0.15$; De la Maza and Parra 1994). Peptides such as mellitin (Ladokhin et al. 1995), magainin (Wieprecht et al. 1999) and PgLA (Wieprecht et al. 2000) forming amphiphilic helices are usually even more active; they can act by detergent-like effects or specific pore formation, depending on conditions.

If membrane permeabilization is initiated by a pair or cluster of surfactin molecules in the membrane, its probability increases non-linearly with $R_{\mathrm{b}}$ regardless of whether the surfactin-surfactin interaction is attractive (dimer formation) or repulsive (driven by thermal energy only). The latter is the rule for membrane-perturbing surfactants (Heerklotz et al. 1994; Keller et al. 1997). There seems to be no reason to assume that surfactin would dimerize (Carrillo et al. 2003) by specific bonds overcompensating the repulsive (including electrostatic) interactions.

\section{Lysis}

The onset of vesicle lysis, i.e., the appearance of first mixed micelles in coexistence with the mixed membranes, is precisely detected by ITC at a mole ratio $R_{\mathrm{b}}^{\mathrm{sat}}=0.22$ in the membrane and a free surfactin concentration of $9 \mu \mathrm{M}$. This interpretation of the ITC data is supported by ${ }^{31} \mathrm{P} \mathrm{NMR}$, revealing an exclusively lamellar powder pattern at $R_{\mathrm{b}}=0.2$, but a small signal indicative of micelles on top of a dominant lamellar spectrum at $R_{\mathrm{b}}=0.3$ (cf. Fig. 5). A more precise detection of $R_{\mathrm{e}}^{\text {sat }}$ by NMR was not pursued since surfactin is not a pure substance and $R_{\mathrm{e}}^{\text {sat }}$ measured at $\sim 120 \mathrm{mM}$ lipid (NMR) may deviate somewhat from the more relevant value at lower concentration obtained by ITC. The minimum mole ratio of surfactinto-lipid in mixed micelles is $R_{\mathrm{m}}^{\mathrm{sol}}=0.43$. Carrillo et al. (2003) observed a drop in light scattering at a total mole ratio of 0.92 that corresponds to either the completion of solubilization or a subsequent transition from large to small micelles (Vinson et al. 1989), but not the onset of solubilization as claimed.

As illustrated by Fig. 2, solubilization is promoted by lower temperature. Whereas $R_{\mathrm{b}}=0.2$ of surfactin in a POPC membrane is not sufficient to induce solubilization at $25^{\circ} \mathrm{C}$, micelles are formed at lower temperature. This can be qualitatively explained by the fact that both thermal disordering of the tails as well as thermal dehydration of the head groups would render the spontaneous curvature of the membrane less positive, counteracting solubilization (Lichtenberg et al. 2005). The thermodynamic background can be illustrated by data published for the surfactant $\mathrm{C}_{12} \mathrm{EO}_{7}$ interacting with POPC (Heerklotz et al. 1997) taking into account that solubilization depends on the balance between micelle formation and membrane partitioning, which yields the approximation $R_{\mathrm{b}}^{\text {sat }} \sim K$.CMC with the partition coefficient $K$ (Heerklotz and Seelig 2000a). $\mathrm{CMC}(T)$ of $\mathrm{C}_{12} \mathrm{EO}_{7}$ exhibits a shallow minimum at $\sim 50^{\circ} \mathrm{C}$ and $K(T)$ a somewhat more pronounced

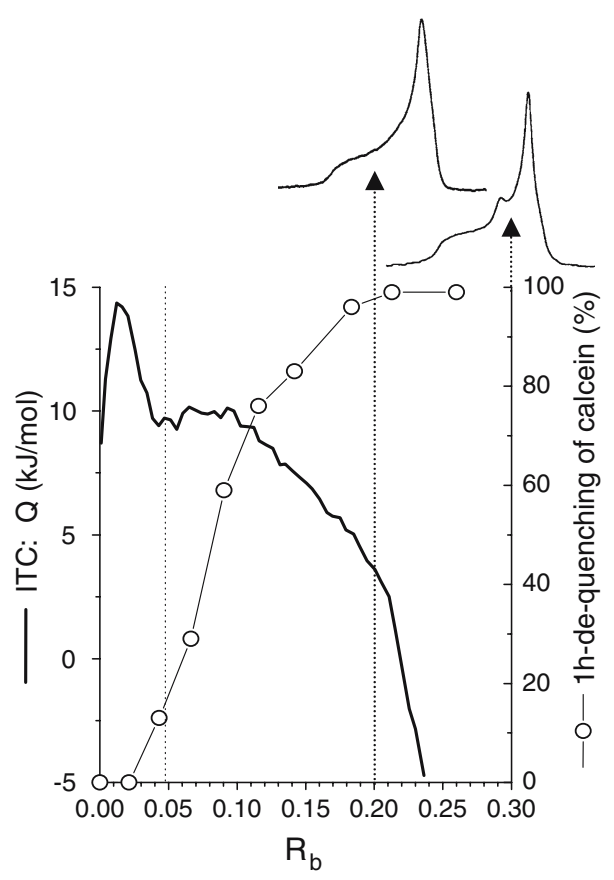

Fig. 5 Combination of ITC (bold line, left axis) and leakage data (open circles, right axis) at $25^{\circ} \mathrm{C}$ and $C_{\mathrm{L}}=1 \mathrm{mM}$, where $C_{\mathrm{S}} /$ $C_{\mathrm{L}} \sim R_{\mathrm{b}} \cdot{ }^{31} \mathrm{P}$ NMR for $R_{\mathrm{b}}=0.3$ but not 0.2 shows evidence for mixed micelles 
maximum at $60^{\circ} \mathrm{C}$. Hence, $R_{\mathrm{b}}^{\text {sat }}(T)$ must increase at least at $50^{\circ} \mathrm{C}(\mathrm{dCMC} / \mathrm{d} T=0, \mathrm{~d} K / \mathrm{d} T>0)$ and at $60^{\circ} \mathrm{C}$ $(\mathrm{dCMC} / \mathrm{d} T>0, \mathrm{~d} K / \mathrm{d} T=0)$. The experimental data reveal that $R_{\mathrm{b}}^{\text {sat }}$ increases with temperature over the whole range from 5 to $80^{\circ} \mathrm{C}$. It should, however, be mentioned that the model discussed here could account also for a decreasing $R_{\mathrm{b}}^{\mathrm{sat}}(T)$ in certain temperature ranges, if the maximum of $K$ is at lower temperature than that of the CMC or if the temperature dependence of $K$ is weaker than that of the CMC.

\section{Membrane-water partitioning}

Knowledge of $R_{\mathrm{b}}$ and the coexisting $C_{\mathrm{S}}^{\mathrm{w}}$ allows calculating the partition coefficient $K$. Taking into account the precision of the different approaches, the data from ITC partitioning experiments $\left(K=22 \mathrm{mM}^{-1}\right.$; Heerklotz and Seelig 2001), from the $C_{\mathrm{L}}$-dependent shift of $R^{\text {sat }}\left(K=24 \mathrm{mM}^{-1}\right.$, Table 1$)$ and from the leakage experiments $\left(K \approx(3 \pm 1) \times 10^{4} \mathrm{M}^{-1}\right.$, Table 1$)$ are fairly consistent. The partition coefficient of surfactin into lipid-saturated mixed micelles is $R_{\mathrm{e}}^{\mathrm{sol} /}$ $C_{\mathrm{S}}^{\mathrm{w}, \mathrm{sol}}=43 \mathrm{mM}^{-1}$, and that into pure surfactin micelles is just $1 / \mathrm{CMC}=133 \mathrm{mM}^{-1}$.

The literature value (Carrillo et al. 2003) of $128 \mathrm{mM}^{-1}$ is essentially in line with these values, taking into account that the respective study was conducted at very high surfactin content, $C_{\mathrm{S}}=5-100 \mu \mathrm{M}$ and $C_{\mathrm{L}}=50 \mu \mathrm{M}$, i.e., predominantly within the mixed micellar range. When the biological properties are concerned, one should of course refer to $K$ as obtained at low concentration in micelle-free systems.

\section{Leakage and antibiotic activity}

There is evidence for the assumption that partitioning of amphiphilic molecules into erythrocyte membranes shows similar partition coefficients as into POPC or EYPC vesicles (Pantaler et al. 2000). Hemolysis by $\mathrm{C}_{15}$-surfactin has an $\mathrm{ED}_{50}$ of $13 \mu \mathrm{M}$ (Kracht et al. 1999). The minimum inhibitory concentration for Acholeplasma laidlawii is $12.5 \mu \mathrm{M}$ (Beven and Wroblewski 1997). The free, aqueous peptide concentration corresponding to an $\mathrm{ED}_{50}$ is expected to be only slightly lower, since the effective lipid concentration in the assays is usually too small (typically $\leq 1 \mathrm{mM}$ ) to bind a major fraction of the peptide. We may conclude that the biologically active concentration of the peptide is of the same order as that inducing destabilization and leakage of POPC vesicles. The induction of an asymmetry between the inner and outer leaflet results in strains and local changes in shape and motility (Beven and Wroblewski 1997), it interferes with endocytosis and exocytosis (Rauch and Farge 2000), and, ultimately, it causes transient membrane failure. Whether these effects suffice to kill the target cell, thus being the relevant mechanism of antibiotic activity, remains to be established.

Acknowledgments We are indebted to Halina Szadkowska and Torsten Wieprecht for valuable technical advice and assistance. Financial support from the Swiss National Science Foundation (Grants \# 31.58800.99 and 3100-067216.01) is gratefully acknowledged.

\section{References}

Allen TM, Cleland LG (1980) Serum-induced leakage of liposome contents. Biochim Biophys Acta 597:418-426

Beven L, Wroblewski H (1997) Effect of natural amphipathic peptides on viability, membrane potential, cell shape and motility of mollicutes. Res Microbiol 148:163-175

Binder H, Lindblom G (2003) Charge-dependent translocation of the Trojan peptide penetratin across lipid membranes. Biophys J 85:982-995

Cameotra SS, Makkar RS (2004) Recent applications of biosurfactants as biological and immunological molecules. Curr Opin Microbiol 7:262-266

Carrillo C, Teruel JA, Aranda FJ, Ortiz A (2003) Molecular mechanism of membrane permeabilization by the peptide antibiotic surfactin. Biochim Biophys Acta Biomembr 1611:91-97

Chellani M (1999) Isothermal titration calorimetry: biological applications. Am Biotechnol Lab 17:14-18

De la Maza A, Parra JL (1994) Vesicle-micelle structural transition of phosphatidylcholine bilayers and Triton $\mathrm{X}$ 100. Biochem J 303:907-914

Dufour S, Deleu M, Nott K, Wathelet B, Thonart P, Paquot M (2005) Hemolytic activity of new linear surfactin analogs in relation to their physico-chemical properties. Biochim Biophys Acta Gen Subj 1726:87-95

Edwards K, Almgren M (1991) Solubilization of lecithin vesicles by $\mathrm{C} 12 \mathrm{E} 8$ - structural transitions and temperature effects. J Colloid Interface Sci 147:1-21

Encinas M, Lissi E (1982) Evaluation of partition constants in compartmentalized systems from fluorescence quenching data. Chem Phys Lett 91:55-57

Heerklotz H (2001) Membrane stress and permeabilization induced by asymmetric incorporation of compounds. Biophys J 81:184-195

Heerklotz H (2002) Triton promotes domain formation in lipid raft mixtures. Biophys J 83:2693-2701

Heerklotz H, Seelig J (2000a) Correlation of membrane/water partition coefficients of detergents with the critical micelle concentration. Biophys J 78:2435-2440

Heerklotz H, Seelig J (2000b) Titration calorimetry of surfactant-membrane partitioning and membrane solubilization. Biochim Biophys Acta 1508:69-85

Heerklotz H, Seelig J (2001) Detergent-like action of the antibiotic peptide surfactin on lipid membranes. Biophys $\mathbf{J}$ 81:1547-1554

Heerklotz H, Binder H, Lantzsch G, Klose G (1994) Membrane/ water partition of oligo (ethylene oxide) dodecyl ethers and its relevance for solubilization. Biochim Biophys Acta 1196:114-122 
Heerklotz H, Lantzsch G, Binder H, Klose G, Blume A (1995) Application of isothermal titration calorimetry for detecting lipid membrane solubilization. Chem Phys Lett 235:517-520

Heerklotz H, Lantzsch G, Binder H, Klose G, Blume A (1996) Thermodynamic characterization of dilute aqueous lipid/ detergent mixtures of POPC and $\mathrm{C} 12 \mathrm{EO} 8$ by means of isothermal titration calorimetry. J Phys Chem 100:67646774

Heerklotz H, Binder H, Lantzsch G, Klose G, Blume A (1997) Lipid/detergent interaction thermodynamics as a function of molecular shape. J Phys Chem B 101:639-645

Heerklotz H, Wieprecht T, Seelig J (2004) Membrane perturbation by the lipopeptide surfactin and detergents as studied by deuterium NMR. J Phys Chem B 108:4909-4915

Hildebrand A, Neubert R, Garidel P, Blume A (2002) Bile saltinduced solubilization of synthetic phosphatidylcholine vesicles studied by isothermal titration calorimetry. Langmuir 18:2836-2847

Kameda Y, Oira S, Matsui K, Kanatomo S, Hase T (1974) Antitumor activity of Bacillus natto. V. Isolation and characterization of surfactin in the culture medium of Bacillus natto KMD 2311. Chem Pharm Bull (Tokyo) 22:938-944

Keller M, Kerth A, Blume A (1997) Thermodynamics of interaction of octyl glucoside with phosphatidylcholine vesicles: partitioning and solubilization as studied by high sensitivity titration calorimetry. Biochim Biophys Acta 1326:178-192

Kinsinger RF, Shirk MC, Fall R (2003) Rapid surface motility in Bacillus subtilis is dependent on extracellular surfactin and potassium ion. J Bacteriol 185:5627-5631

Kracht M, Rokos H, Ozel M, Kowall M, Pauli G, Vater J (1999) Antiviral and hemolytic activities of surfactin isoforms and their methyl ester derivatives. J Antibiot (Tokyo) 52:613-619

Ladokhin AS, White SH (2001) 'Detergent-like' permeabilization of anionic lipid vesicles by melittin. Biochim Biophys Acta 1514:253-260

Ladokhin AS, Wimley WC, White SH (1995) Leakage of membrane vesicle contents: determination of mechanism using fluorescence requenching. Biophys J 69:1964-1971

Lange Y, Dolde J, Steck TL (1981) The rate of transmembrane movement of cholesterol in the human erythrocyte. J Biol Chem 256:5321-5323

Lichtenberg D, Robson RJ, Dennis EA (1983) Solubilization of phospholipids by detergents. Structural and kinetic aspects. Biochim Biophys Acta 737:285-304

Lichtenberg D, Goni FM, Heerklotz H (2005) Detergentresistant membranes should not be identified with membrane rafts. Trends Biochem Sci 30:430-436

Lohner K, Epand R (1997) Membrane interactions of hemolytic and antibacterial peptides. Adv Biophys Chem 6:53-66

MacDonald RC, MacDonald RI, Menco BP, Takeshita K, Subbarao NK, Hu LR (1991) Small-volume extrusion apparatus for preparation of large, unilamellar vesicles. Biochim Biophys Acta 1061:297-303

Pantaler E, Kamp D, Haest CW (2000) Acceleration of phospholipid flip-flop in the erythrocyte membrane by detergents differing in polar head group and alkyl chain length. Biochim Biophys Acta 1509:397-408

Paternostre MT, Roux M, Rigaud JL (1988) Mechanisms of membrane protein insertion into liposomes during reconstitution procedures involving the use of detergents. 1 . Solubilization of large unilamellar liposomes (prepared by reverse-phase evaporation) by triton X-100, octyl glucoside, and sodium cholate. Biochemistry 27:2668-2677

Prenner EJ, Lewis RN, Jelokhani-Niaraki M, Hodges RS, McElhaney RN (2001) Cholesterol attenuates the interaction of the antimicrobial peptide gramicidin $\mathrm{S}$ with phospholipid bilayer membranes. Biochim Biophys Acta 1510:83-92

Rauch C, Farge E (2000) Endocytosis switch controlled by transmembrane osmotic pressure and phospholipid number asymmetry. Biophys J 78:3036-3047

Sheetz MP, Singer SJ (1974) Biological membranes as bilayer couples. A molecular mechanism of drug-erythrocyte interactions. Proc Natl Acad Sci USA 71:4457-4461

Vinson PK, Talmon Y, Walter A (1989) Vesicle-micelle transition of phosphatidylcholine and octyl glucoside elucidated by cryo-transmission electron-microscopy. Biophys J 56:669-681

Vollenbroich D, Ozel M, Vater J, Kamp RM, Pauli G (1997a) Mechanism of inactivation of enveloped viruses by the biosurfactant surfactin from Bacillus subtilis. Biologicals 25:289-297

Vollenbroich D, Pauli G, Ozel M, Vater J (1997b) Antimycoplasma properties and application in cell culture of surfactin, a lipopeptide antibiotic from Bacillus subtilis. Appl Environ Microbiol 63:44-49

Wenk MR, Seelig J (1997) Vesicle-micelle transformation of phosphatidylcholine/octyl-beta-D-glucopyranoside mixtures as detected with titration calorimetry. J Phys Chem B 101:5224-5231

Wieprecht T, Dathe M, Epand RM, Beyermann M, Krause E, Maloy WL, MacDonald DL, Bienert M (1997) Influence of the angle subtended by the positively charged helix face on the membrane activity of amphipathic, antibacterial peptides. Biochemistry 36:12869-12880

Wieprecht T, Beyermann M, Seelig J (1999) Binding of antibacterial magainin peptides to electrically neutral membranes: thermodynamics and structure. Biochemistry 38:10377-10387

Wieprecht T, Apostolov O, Beyermann M, Seelig J (2000) Membrane binding and pore formation of the antibacterial peptide PGLa: thermodynamic and mechanistic aspects. Biochemistry 39:442-452 